\title{
Effect of Ultrasonography-Guided Botulinum Toxin Type A Injection in Holmes' Tremor Secondary to Pontine Hemorrhage: Case Report
}

\author{
So-Yeon Ahn, $\mathrm{MD}^{1}$, Dong-A Kim, MD, PhD ${ }^{1}$, Youn-Ok Park, $\mathrm{MS}^{2}$, Joon-Ho Shin, $\mathrm{MD}^{1}$ \\ ${ }^{1}$ Department of Rehabilitation Medicine, Rehabilitation Hospital, National Rehabilitation Center, Seoul; \\ ${ }^{2}$ Division of Education \& Public Relations, National Rehabilitation Center, Seoul, Korea
}

Holmes' tremor is a low-frequency rest and intentional tremor secondary to various insults, including cerebral ischemia, hemorrhage, trauma, or neoplasm. Pharmacologic treatment is usually unsuccessful, and some cases require surgical intervention. We report a rare case of Holmes' tremor secondary to left pontine hemorrhage in a 29-year-old Asian male patient who developed 1.6-Hz postural and rest tremor of the right hand. He responded markedly to ultrasonography-guided botulinum toxin type A injection. To our knowledge, this is the first report of Homes' tremor treated with ultrasonography-guided botulinum toxin type A injection with favorable results.

Keywords Tremor, Botulinum toxins, Ultrasonography

\section{INTRODUCTION}

Holmes' tremor was first described in 1904 [1]; it is characterized as both a rest and intentional tremor that is often non-rhythmical [2]. The tremor is of low-frequency flexion-extension oscillation at rest, mostly below 4.5 $\mathrm{Hz}$, exacerbated by posture and action, and generally unilateral [1-3]. There is variable delay (approximately 4 weeks to 2 years) between the lesion and the first occurrence of tremor [1-3]. Treatment includes oral drugs,

Received May 28, 2013; Accepted October 28, 2013

Corresponding author: Joon-Ho Shin

Department of Rehabilitation Medicine, Rehabilitation Hospital, National Rehabilitation Center, 58 Samgaksan-ro, Gangbuk-gu, Seoul 142-884, Korea

Tel: +82-2-901-1884, Fax: +82-2-902-3835, E-mail: asfreelyas@gmail.com

(c) This is an open-access article distributed under the terms of the Creative Commons Attribution Non-Commercial License (http://creativecommons. org/licenses/by-nc/3.0) which permits unrestricted noncommercial use, distribution, and reproduction in any medium, provided the original work is properly cited.

Copyright (c) 2014 by Korean Academy of Rehabilitation Medicine such as levodopa, dopamine agonists, propranolol, clonazepam, and levetiracetam; thalamic surgery; and deep brain stimulation. However, such treatments have been described only by isolated case reports, thus treatment remains challenging.

We report a patient with Holmes' tremor secondary to pontine hemorrhage previously diagnosed with schizophrenia and failed usual medical treatment. The patient responded markedly well to ultrasonography-guided botulinum toxin type A (BoNT-A) injection.

\section{CASE REPORT}

A 29-year-old Asian male patient with a 4-month history of $1.6-\mathrm{Hz}$ postural and rest tremor of the right hand, consistent with Holmes' tremor, visited our department. He had been quadriplegic secondary to left pontine hemorrhage for 6 months (Fig. 1). His medical history was notable for a 6-year history of paranoid-type schizophrenia 

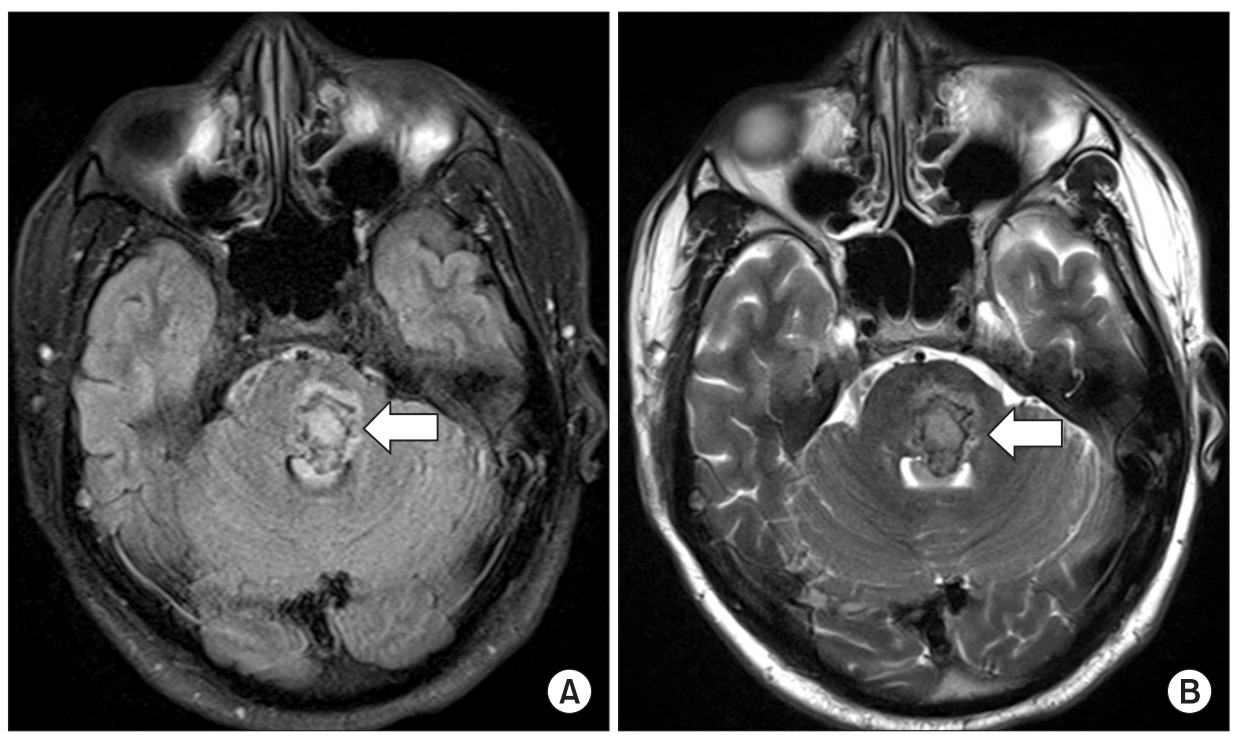

Fig. 1. (A) Axial fluid-attenuated inversion recovery and (B) T2weighted magnetic resonance imaging scans (inset) showing acute intracerebral hemorrhage in the left pons (arrow).

and psychiatric treatment with several typical and atypical antipsychotic agents. Four months ago, a 1.4- to $2-\mathrm{Hz}$ tremor developed in his right hand and gradually spread to the wrist and elbow, characterized by irregular flexionextension oscillation, distal prominence, presence at rest, and increase with posture. It worsened his right upper extremity function and his gait, leading to limitation of activities of daily living. In family history, his mother had schizophrenia but there was no history of movement disorders, including tremors and other neurologic illnesses.

Current medications were olanzapine (10 mg daily), amisulpride (100 mg daily), and lorazepam ( $0.5 \mathrm{mg}$ daily) as antipsychotic agents for the past 6 years, and levetiracetam (1,000 $\mathrm{mg}$ daily) as seizure prophylaxis for the past 6 months. For tremor relief, he received clonazepam (4 mg daily) for 3 months and levodopa (500 mg daily) for 2 months. Although these medications mildly improved the tremor, visual hallucinations became more vivid and frequent.

Therefore, we administered 100 units (U) of BoNT-A (Botox; Allergan, Irvine, CA, USA) while decreasing the dosages of the medications for tremor. Injection was performed by an expert physiatrist using ultrasonography (ACCUVIX XG; Medison, Seoul, Korea) interfaced with a 5-13 MHz linear array transducer and electrical stimulation (Stimuplex; B. Braun Melsungen AG, Melsungen, Germany) guidance in the right side of the flexor digitorum superficialis of the second, third, and fourth digits (20 U each), and in the extensor pollicis longus (40 U) muscles (Fig. 2). Those muscles were selected because

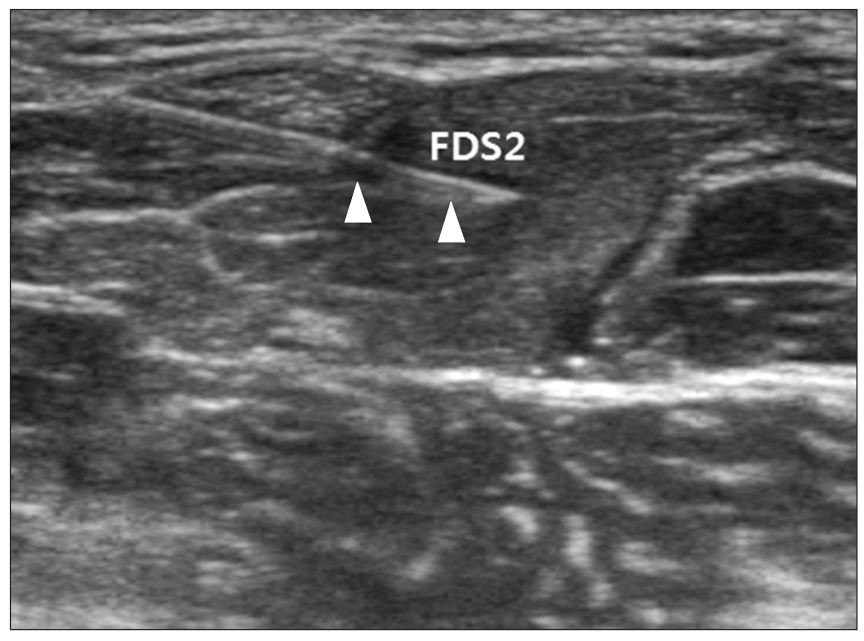

Fig. 2. Ultrasonographic image of the right second flexor digitorum superficialis (FDS2). The needle (arrowheads) is advanced into the FDS2 under real-time ultrasonography guidance.

they showed the most vivid contraction on real-time ultrasonography during rest and during provoked state by clenching his left fist and elevating his lower extremities. During the first few days after infiltration, improvement of symptoms, mainly tremor on extension of the first, second, and third fingers, was observed. Rest tremors showed marked improvement, and postural and intentional tremors also improved, although to a lesser extent.

The frequency of tremor was measured retrospectively with video recording of ultrasonography; it decreased from $1.4-2 \mathrm{~Hz}$ to $0-0.5 \mathrm{~Hz}$. In terms of activities of daily 
living, improvements in eating and dressing were observed. After 1 month of injections, he could perform small object manipulation, which was not possible at admission. The maximum benefit became evident approximately 2 weeks later and lasted for 2 months. Then, rest tremor reoccurred progressively. After 3 months of BoNT-A injections, the frequency of tremor decreased from $1.4-2 \mathrm{~Hz}$ to $1-1.4 \mathrm{~Hz}$, and the duration at provoked state shortened from 3-5 to 2-3 minutes. Injection of 100 $\mathrm{U}$ of BoNT-A was repeated in the same muscles using ultrasonography guidance.

\section{DISCUSSION}

Holmes' tremor is a rare late effect of lesions that occur in the brainstem, cerebellum, or thalamus owing to various nigrostriatal and cerebellothalamic pathwaydamaging insults, including cerebral ischemia, hemorrhage, trauma, or neoplasm [2,4]. In most cases, medical therapy shows poor response, although levetiracetam, levodopa, clonazepam, ventralis intermedius nucleus thalamotomy, and deep brain stimulation have been reported to be effective in several case reports $[3,5,6]$. Alternative medical or surgical approaches for Holmes' tremor are not yet well established because of the absence of controlled studies. Unlike drug-induced tremor, the tremor in this case was nonrhythmical, with lowfrequency (mostly 1.4-2 Hz) flexion-extension oscillation at rest [7]. Despite using the same antipsychotics for 6 years, no movement complaints, such as tremor, were ever reported. Tremor only occurred 2 months after pontine hemorrhage.

Recently, BoNT-A was recommended by 2 class II studies as a treatment option for essential hand tremor that has failed with other pharmacologic treatments [8]. In this case, BoNT-A injections improved the rest tremor considerably and the postural and intentional tremors mildly in a patient in whom long-term multiple pharmacological treatments had been unsuccessful. In this patient, especially because of his schizophrenia, we could not adequately increase the dose of medication and had to use a dopamine antagonist at the same time. Therefore, BoNT-A could have been more beneficial, considering the localized effect.

Ultrasonography has proved its validity and reliability as a diagnostic imaging tool for muscles and nerves [9]. We performed ultrasonography-guided BoNT injection in the upper limb muscles, which permitted accurate muscle selection. This procedure has been suggested as a standard technique for deep-seated and small muscles [10]. In addition, ultrasonography allowed us to compare tremor using frequency of muscle contraction as an objective indicator before and after treatment to evaluate progression. Frequency was obtained by counting each muscle contraction with the use of retrospective video recording analysis. To our knowledge, this is the first case of tremor evaluated using ultrasonography. Clinical numeric rating scales or ataxia scales also can be used for tremor evaluation, but they tend to be underestimated in subjects who have paralysis, spasticity, or decreased motor power. Although ultrasonography does not evaluate neurophysiological properties, only anatomic aspects, it has the advantages of noninvasiveness and easy accessibility. It also enables real-time inspection during injection and video recording.

Despite the transient effect of BoNT-A, it was the treatment of choice in this case due to the prescription limitations for the patient because of his schizophrenia. He also did not want an invasive treatment, such as surgery. The results in our case support the potential of BoNT-A injection in patients with Holmes' tremor before applying more invasive therapies. In addition, ultrasonography can be a useful instrument for the objective evaluation and treatment of tremor.

\section{CONFLICT OF INTEREST}

No potential conflict of interest relevant to this article was reported.

\section{ACKNOWLEDGMENTS}

We thank Professor Sang Yun Kim (Department of Neurology, Seoul National University College of Medicine \& Seoul National University Bundang Hospital) for helping in the diagnosis and sharing his insights. This study was supported by a grant from the Ministry of Trade, Industry \& Energy (MOTIE) and National IT Industry Promotion Agency (NIPA) in the SW Convergence Program for Demonstration and Diffusion 2012.

\section{REFERENCES}

1. Holmes G. On certain tremors in organic cerebral le- 
sions. Brain 1904;27:327-75.

2. Deuschl G, Bergman H. Pathophysiology of nonparkinsonian tremors. Mov Disord 2002;17 Suppl 3:S41-8.

3. Acar G, Acar F, Bir LS, Kizilay Z, Cirak B. Vim stimulation in Holmes' tremor secondary to subarachnoid hemorrhage. Neurol Res 2010;32:992-4.

4. Seidel S, Kasprian G, Leutmezer F, Prayer D, Auff E. Disruption of nigrostriatal and cerebellothalamic pathways in dopamine responsive Holmes' tremor. J Neurol Neurosurg Psychiatry 2009;80:921-3.

5. Kim MC, Son BC, Miyagi Y, Kang JK. Vim thalamotomy for Holmes' tremor secondary to midbrain tumour. J Neurol Neurosurg Psychiatry 2002;73:453-5.

6. Striano P, Elefante A, Coppola A, Tortora F, Zara F, Minetti C, et al. Dramatic response to levetiracetam in post-ischaemic Holmes' tremor. J Neurol Neurosurg Psychiatry 2007;78:438-9.
7. Zeuner KE, Deuschl G. An update on tremors. Curr Opin Neurol 2012;25:475-82.

8. Simpson DM, Blitzer A, Brashear A, Comella C, Dubinsky R, Hallett $\mathrm{M}$, et al. Assessment: Botulinum neurotoxin for the treatment of movement disorders (an evidence-based review): report of the Therapeutics and Technology Assessment Subcommittee of the American Academy of Neurology. Neurology 2008;70:1699-706.

9. Cartwright MS, Demar S, Griffin LP, Balakrishnan N, Harris JM, Walker FO. Validity and reliability of nerve and muscle ultrasound. Muscle Nerve 2013;47:515-21.

10. Wissel J, Ward AB, Erztgaard P, Bensmail D, Hecht MJ, Lejeune TM, et al. European consensus table on the use of botulinum toxin type A in adult spasticity. J Rehabil Med 2009;41:13-25. 